\title{
Damped-driven granular chains: An ideal playground for dark breathers and multibreathers
}

\author{
C. Chong, ${ }^{1, *}$ F. Li,,${ }^{2, \dagger}$ J. Yang, ${ }^{3}$ M. O. Williams,${ }^{4}$ I. G. Kevrekidis, ${ }^{4}$ P. G. Kevrekidis, ${ }^{1}$ and C. Daraio ${ }^{2,5}$ \\ ${ }^{1}$ Department of Mathematics and Statistics, University of Massachusetts, Amherst, Massachusetts 01003-4515, USA \\ ${ }^{2}$ Graduate Aerospace Laboratories (GALCIT), California Institute of Technology, Pasadena, California 91125, USA \\ ${ }^{3}$ Aeronautics and Astronautics, University of Washington, Seattle, Washington 98195-2400, USA \\ ${ }^{4}$ Department of Chemical and Biological Engineering and PACM, Princeton University, Princeton, New Jersey 08544, USA \\ ${ }^{5}$ Department of Mechanical and Process Engineering (D-MAVT), Swiss Federal Institute of Technology (ETH), 8092 Zurich, Switzerland
}

(Received 17 July 2013; revised manuscript received 3 February 2014; published 31 March 2014)

\begin{abstract}
By applying an out-of-phase actuation at the boundaries of a uniform chain of granular particles, we demonstrate experimentally that time-periodic and spatially localized structures with a nonzero background (so-called dark breathers) emerge for a wide range of parameter values and initial conditions. We demonstrate a remarkable control over the number of breathers within the multibreather pattern that can be "dialed in" by varying the frequency or amplitude of the actuation. The values of the frequency (or amplitude) where the transition between different multibreather states occurs are predicted accurately by the proposed theoretical model, which is numerically shown to support exact dark breather and multibreather solutions. Moreover, we visualize detailed temporal and spatial profiles of breathers and, especially, of multibreathers using a full-field probing technology and enable a systematic favorable comparison among theory, computation, and experiments. A detailed bifurcation analysis reveals that the dark and multibreather families are connected in a "snaking" pattern, providing a roadmap for the identification of such fundamental states and their bistability in the laboratory.
\end{abstract}

DOI: 10.1103/PhysRevE.89.032924

PACS number(s): 05.45.-a, 45.70.-n, 63.20.Ry, 63.20.Pw

\section{INTRODUCTION}

The study of discrete breathers has been a topic of intense theoretical and experimental interest during the 25 years since their theoretical inception, as has been recently summarized, e.g., in [1]. Among the broad and diverse list of fields where such time-periodic structures that are exponentially localized in space have been of interest, we mention, for instance, optical waveguide arrays or photorefractive crystals [2], micromechanical cantilever arrays [3], Josephson-junction ladders [4], layered antiferromagnetic crystals [5], halidebridged transition metal complexes [6], dynamical models of the DNA double-strand [7], and Bose-Einstein condensates in optical lattices [8]. However, most of these investigations have been restricted to the context of bright such states, namely, ones supported on a vanishing background. Dark breathers (DBs), i.e., breather states on a nonvanishing background, have been far less widely studied. Their recent realization in contexts such as surface water waves [9], Bose-Einstein condensates [10] (see also [11] for a recent review), ferromagnetic film strips [12], or optical waveguide arrays (for a recent example see, e.g., [13], and references therein) has received considerable attention. Note, however, that the DBs reported on in these works are based on the observation of dark (envelope) solitary waves. To the best of our knowledge, there are no results on the experimental observation of genuinely time-periodic DBs.

Granular chains, which consist of closely packed arrays of particles $[14,15]$ that interact elastically, are relevant for numerous applications such as shock and energy absorbing layers [16-19], actuating devices [20], acoustic lenses [21], acoustic diodes [22], and sound scramblers [23,24]. At a fundamental level, granular chains have been shown to

\footnotetext{
*chong@math.umass.edu

${ }^{\dagger}$ Corresponding author: fengli@ caltech.edu
}

support defect modes [25], bright discrete breathers in dimer chains (i.e., bearing two alternating masses) [26], and surface variants thereof [27]. Such bright breathers have been detected experimentally by placing force sensors at isolated particles within the granular chain. We note that with this measurement technique a full-field representation of the breather is not possible. Very recently, DBs were theoretically proposed in a Hamiltonian variant of the system as the sole discrete breather configuration that can arise in a "monoatomic" chain, i.e., a chain where all the particles are identical [28].

The main result of the present work is the full-field visualization, in both the spatial and the temporal domains, of DBs (and their multibreather generalizations) in a monoatomic granular chain. We are able to observe the structures over several hundred periods of motion, demonstrating their spatially localized and time-periodic nature. By considering a realistic damped-driven model for the monoatomic granular chain, we are able to predict the type of breather that will emerge, be it a DB or a multibreather. Thus, one has remarkable control over breather formation by tuning the system parameters accordingly. The resulting bifurcation diagram consists of a single coiling branch (often referred to as "snaking") and compares well with the experiments. The paper is organized as follows: In Secs. II and III we describe the experimental and theoretical setups, respectively. The main results are presented in Sec. IV, whereas Sec. V details the linear stability analysis. In Sec. VI a deeper theoretical probing of the bifurcation scenario is carried out, and conclusions and future challenges are given in Sec. VII.

\section{EXPERIMENTAL SETUP}

A DB is a time-periodic structure with tails that oscillate at a finite amplitude (as opposed to bright breathers, where the oscillation amplitude asymptotes to 0 as the lattice index $n \rightarrow \infty$ ); see, e.g., Fig. 1. For example, the function 

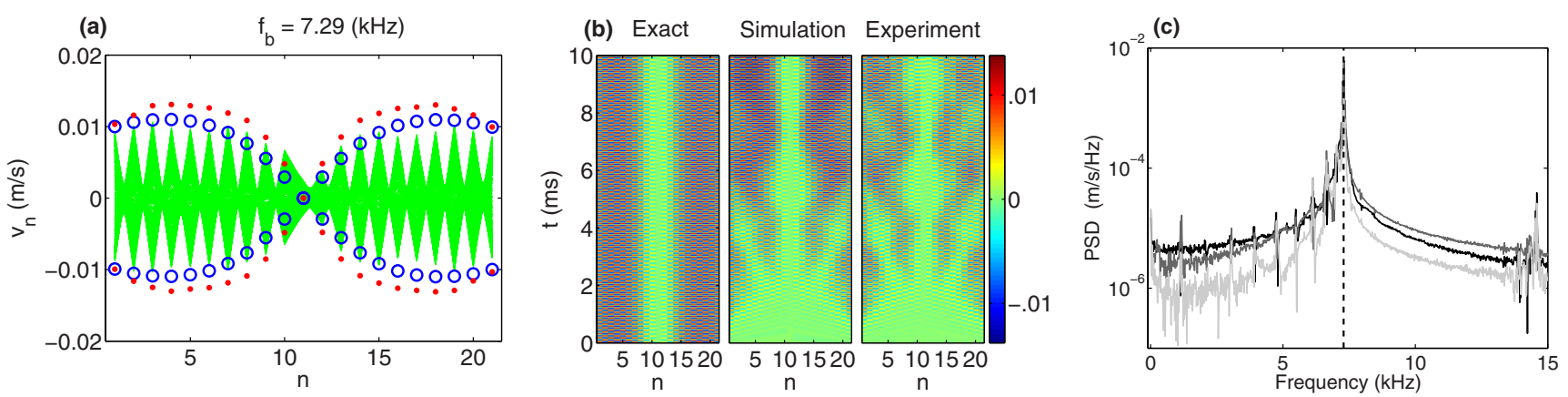

FIG. 1. (Color online) (a) Experimentally measured velocities versus bead number for $f_{b}=7.29 \mathrm{kHz}$ and $a=0.2 \mu \mathrm{m}$. The entire time series of each bead location is shown as a superposition [shaded (green) central areas]. Extrema as predicted by the simulation for the first $10 \mathrm{~ms}$ [(red) points] and numerically exact dark breather [open (blue) circles] are also shown. (b) Space-time contour plots of the exact (numerically obtained) breather, a transiently simulated one from zero initial data, and the corresponding experimental evolution, also from zero initial data, leading to the same state. Color intensity corresponds to velocity $(\mathrm{m} / \mathrm{s})$. (c) Experimentally measured power spectral density $(\mathrm{m} / \mathrm{s} / \mathrm{Hz})$ of bead 1 (black line), bead 5 (dark-gray line), and bead 10 (light-gray line) for an actuation amplitude of $a=0.2 \mu \mathrm{m}$. The dashed vertical line corresponds to the driving frequency $f_{b}=7.29 \mathrm{kHz}$.

$(-1)^{n} \alpha \tanh (\beta n) \cos \left(2 \pi f_{b} t\right)$, with $\alpha$ and $\beta$ constants, can be thought of as a DB with frequency $f_{b}$. DBs were shown to have this form in several nonlinear lattice models including the Klein-Gordon lattice [30], the Fermi-Pasta-Ulam lattice $[31,32]$, and the (Hamiltonian) monoatomic granular crystal lattice [28]. Following the theoretical proposal in [28], we intend to use a destructive interference mechanism to spontaneously generate DBs. We actuate the granular crystal at both of its boundaries at frequency $f_{b}$, i.e., the frequency of our intended DB. In order to induce a vanishing amplitude at the central site (and the density dip associated with a DB), there should be an odd number of beads and out-of-phase actuation such that incoming waves from each boundary actuation will cancel each other out at the center.

Figure 2 shows the schematic of the experimental setup consisting of a 21-sphere granular chain and a laser Doppler vibrometer (Polytec OFV-534). The spheres have a radius $R=$ $9.53 \mathrm{~mm}$ and are made of chrome steel (with Young's modulus $E=200 \mathrm{GPa}$, Poisson ratio $v=0.3$, and mass $M=28.2 \mathrm{~g}$ ) [33]. They are supported by four polytetrafluoroethylene rods allowing free axial vibrations of the particles, while restricting

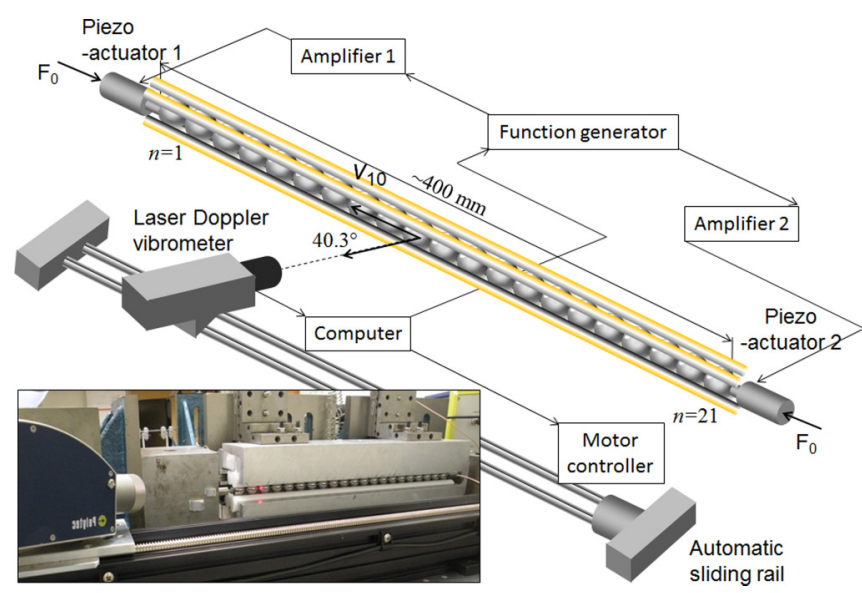

FIG. 2. (Color online) Schematic of the experimental setup. Inset: Digital image of the setup. their lateral motions. Both ends of the granular chain are compressed with a static force $\left(F_{0}=10 \mathrm{~N}\right.$, as in [34]), and they are driven by two piezoelectric actuators that are powered individually by an external function generator and two amplifiers. The laser Doppler vibrometer measures the individual particles' velocity profiles and produces a full-field map of the granular chain dynamics.

\section{THE DAMPED-DRIVEN MODEL}

To model the experimental setup we incorporate into the standard granular crystal model [14] a simple description of the dissipation [22] and out-of-phase actuators on the left and right boundaries,

$$
\begin{aligned}
M \ddot{u}_{n}= & A\left[\delta_{0}+u_{n-1}-u_{n}\right]_{+}^{3 / 2} \\
& -A\left[\delta_{0}+u_{n}-u_{n+1}\right]_{+}^{3 / 2}-\frac{M}{\tau} \dot{u}_{n}, \\
u_{0}= & a \cos \left(2 \pi f_{b} t\right), \quad u_{N+1}=-a \cos \left(2 \pi f_{b} t\right),
\end{aligned}
$$

where $N$ (odd) is the number of beads in the chain, $u_{n}(t)$ is the displacement of the $n$th bead from the equilibrium position at time $t, A=\frac{E \sqrt{2 R}}{3\left(1-v^{2}\right)}, M$ is the bead mass, and $\delta_{0}$ is an equilibrium displacement induced by a static load $F_{0}=A \delta_{0}^{3 / 2}$. The bracket is defined by $[x]_{+}=\max (0, x)$. The strength of the dissipation is captured by the parameter $\tau$, whereas $a$ and $f_{b}$ represent the amplitude and frequency of the actuation, respectively. In what follows, we fix $\tau=5 \mathrm{~ms}$ based on experimental observation (see the Appendix) and treat $a$ and $f_{b}$ as the sole control parameters.

The pass band of the linearized equations of motion is $\left[0, f_{0}\right]$, where $f_{0}=\sqrt{\frac{3 A}{2 M \pi^{2}}} \delta_{0}^{1 / 4}$ is the cutoff frequency. For the parameter values used herein, $f_{0}=7.373 \mathrm{kHz}$. To obtain DBs experimentally (and in numerical simulations) we use the following excitation procedure: the frequency of actuation is chosen within the pass band $f_{b} \in\left[0, f_{0}\right]$ with zero initial conditions. In this case, the propagation of plane waves and their subsequent destructive interference spontaneously produces the DBs. In order to ensure the robust formation of a DB and avoid the onset of transient, high-amplitude traveling 


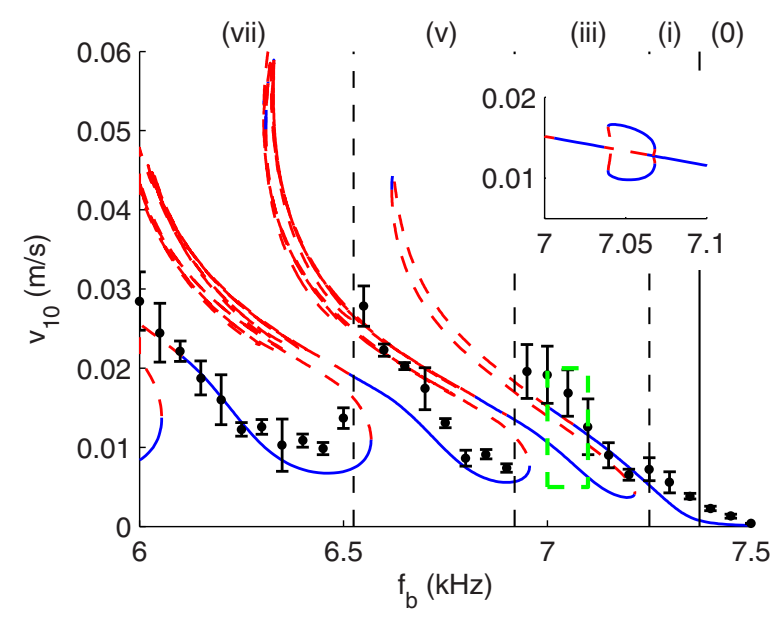

FIG. 3. (Color online) Bifurcation diagram illustrating the various dark breather/multibreather branches. Specifically, the maximum velocity of bead 10 versus the frequency $f_{b}$ is shown with $a=0.2 \mu \mathrm{m}$. Smooth curves correspond to numerically exact dark breathers; solid (blue) lines indicate the absence of a real instability, and dashed (red) lines indicate their presence (see Sec. V). The linear cutoff frequency $f_{0}=7.373 \mathrm{kHz}$ is indicated by the solid vertical black line. Black circles represent the experimentally measured mean values (with standard deviations given by the error bars) which were obtained using four experimental runs. Inset: Zoom-in of the dashed (green) box showing a representative example of additional branches that emerge from (and disappear back into) the main branch.

waves [14], we tune the actuation amplitude to be increased linearly from 0 to the desired amplitude $a$ over some fixed number of periods (we chose eight).

DBs obtained with this excitation procedure are compared with numerically exact (up to a prescribed tolerance) periodic solutions of Eq. (1) of period $T_{b}$ by computing roots of the map $F=u\left(T_{b}\right)-u(0)$, where $u(0)$ is the initial-state vector of the (numerically realized) stroboscopic map and $u\left(T_{b}\right)$ is the solution of the equations of motion at time $T_{b}$. The Jacobian of $F$, which is used in the Newton iterations, is of the form $V\left(T_{b}\right)-I$, where $I$ is the identity matrix, $V$ is the solution to the variational equation $V^{\prime}=D F \cdot V$ with initial condition $V(0)=I$, and $D F$ is the Jacobian of the equations of motion evaluated at $u$. Note that the breather frequency and actuation frequency are both $f_{b}=1 / T_{b}$ by construction.

\section{MAIN RESULTS}

Figures 1(a) and 1(b) show numerical and experimental DB excitation for the "typical" values of $f_{b}=7.29 \mathrm{kHz}$ and $a=0.2 \mu \mathrm{m}$ and the corresponding numerically exact solution at those parameter values. The strong structural similarity in space to an exact DB [see, e.g., Fig. 1(a)] suggests that both are near the steady state after $10 \mathrm{~ms}$. It is not surprising, then, that the motion of the beads is periodic, as shown by the power spectral density plot [Fig. 1(c)]. The detailed comparison of the space-time evolution of the numerically exact solution, simulation, and experiment in Fig. 1(b) shows that after the initial transient stage of the dynamics, a DB is formed. We note that the maximum strain $\left|u_{n}-u_{n+1}\right|$ of the solution is about $60 \%$ of the precompression, confirming that the structures reported here are a result of the nonlinearity of the system and that any analysis based on the Taylor expansion of the nonlinearity (including, e.g., the derivation of the nonlinear Schrödinger equation $[28,29]$ ) is not relevant in our setup.

We now study the full bifurcation diagram of the DBs shown in Fig. 3 and the corresponding profiles/evolutions illustrated in Fig. 4. Numerical continuation reveals that, for a fixed driving amplitude (here, $a=0.2 \mu \mathrm{m}$ ), the DBs and multibreathers appear to be located on a single coiling solution branch. This structure, sometimes referred to as "snaking" in the dynamical systems community [35-41], has received considerable recent attention in settings such as nematic liquid crystals [42] and classical fluid problems such as Couette flow [43]. To the best of the authors' knowledge, snaking behavior in FPU-like chains (such as a granular crystal chain) has not been reported previously. Each fold represents the collision of two breather families, such as a DB with a multibreather. Due to the "coiling" structure and the nature of excitation simulations and experiments considered herein (which start with a zero initial state), the transition from one multibreather family to the next as the forcing frequency is increased (or decreased) is not smooth: saddle-node bifurcations cause the solution to "jump" from lobe to lobe. For example, in Fig. 3 the regions labeled (vii), (v), (iii), and (i) correspond, respectively, to the number of density dips seen in the (experimental) space-time contour
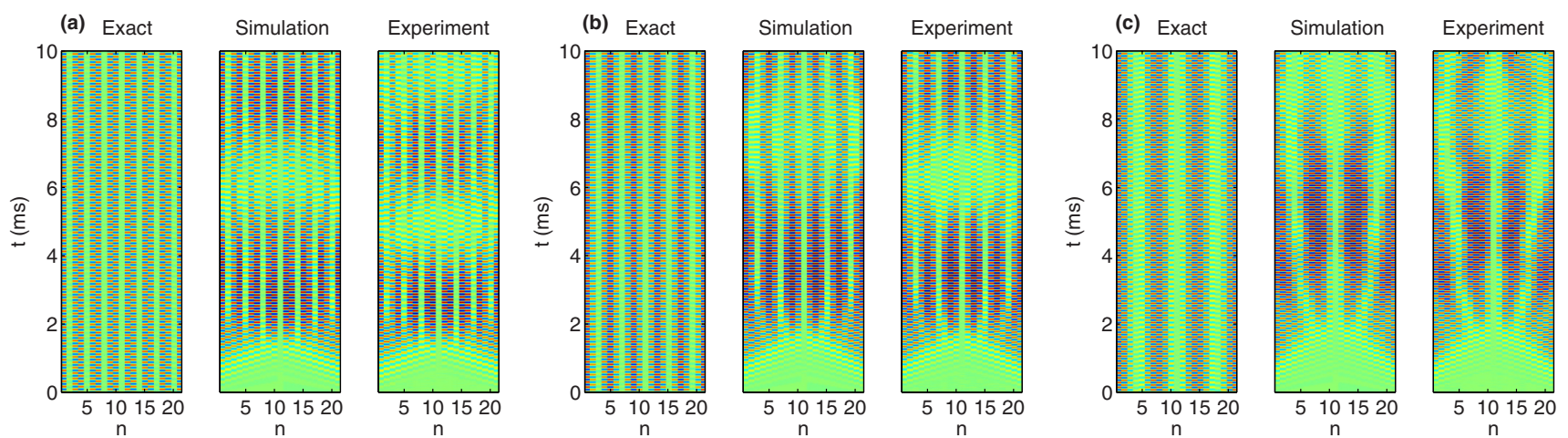

FIG. 4. (Color online) Space-time contour plots of the numerically exact, simulated, and experimental evolution leading to a dark breather with $a=0.2 \mu \mathrm{m}$. (a) Seven-dip solution with $f_{b}=6.35 \mathrm{kHz}$. (b) Five-dip solution with $f_{b}=6.8 \mathrm{kHz}$. (c) Three-dip solution with $f_{b}=$ $7.15 \mathrm{kHz}$. Color intensity corresponds to velocity $(\mathrm{m} / \mathrm{s})$; the color legend in each panel is the same as in Fig. 1. 
(a) $\mathrm{f}_{\mathrm{b}}=7.14(\mathrm{kHz})$

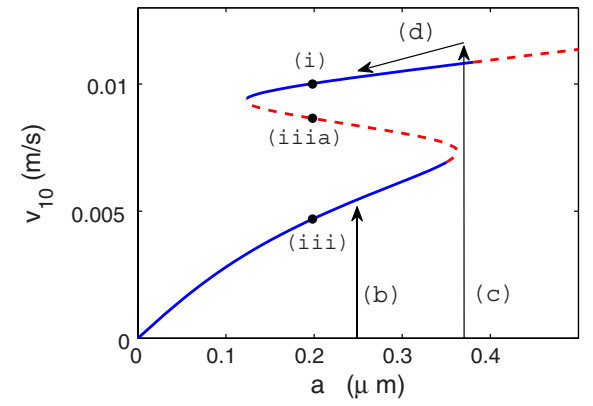

(b) $\quad \mathrm{a}=0.25(\mu \mathrm{m})$

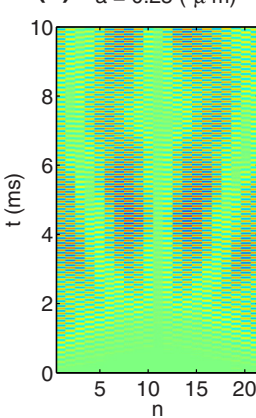

(c) $\mathrm{a}=0.37(\mu \mathrm{m})$

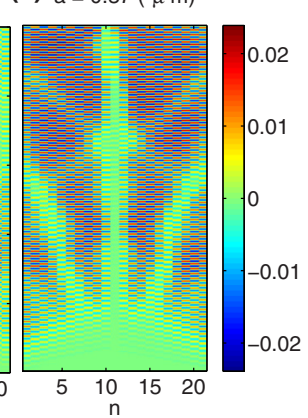

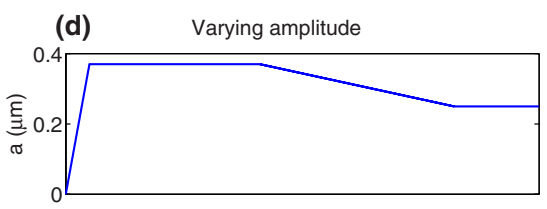

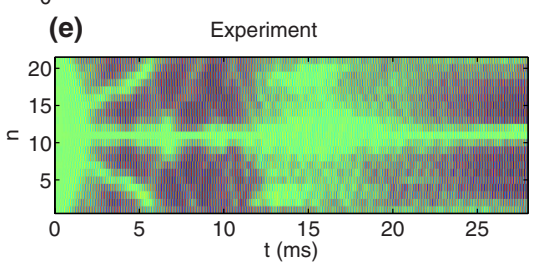

FIG. 5. (Color online) Experimental observation of bistability. (a) Maximum velocity of bead 10 of the exact dark breather versus actuation amplitude $a$ with the frequency fixed as $f_{b}=7.14 \mathrm{kHz}$. Black points indicate the value $a=0.2 \mu \mathrm{m}$, which corresponds to continuation with respect to the frequency shown in Fig. 3. One can see that the single-dip breather (i) and three-dip breather (iii) are connected by an intermediate unstable solution (iiia). Arrows labeled (b)-(d) describe how the bistability of the system can be realized experimentally [see (b)-(d)]. (b) Experimental space-time contour plots of the velocity. By choosing the actuation amplitude $a$ appropriately one can excite a three-dip breather $(a=0.25 \mu \mathrm{m})$. (c) Same as (b), but for an actuation amplitude that dials in a single-dip breather $(a=0.37 \mu \mathrm{m})$. Note that the three-dip breather terminates at $a \approx 0.36 \mu \mathrm{m}$ in (a). (d) Actuation amplitude profile used to excite a high-energy dark breather. After the initial linear ramping, the amplitude is kept at $a=0.37 \mu \mathrm{m}$ for about $10 \mathrm{~ms}$, which will excite a single-dip breather. The single-dip breather is still maintained even when decreasing the amplitude to $a=0.25 \mu \mathrm{m}$. (e) Space-time contour plot corresponding to the actuation amplitude profile shown in (d). Color intensity corresponds to velocity $(\mathrm{m} / \mathrm{s})$, where the color bar is the same as in (c). Note that if one actuates a resting chain with an actuation amplitude of $a=0.25 \mu \mathrm{m}$, then a three-dip breather will emerge [see (b)], thus revealing the system's bistability.

plot. The label (0) corresponds to the breathers localized near the boundaries rather than at the center of the domain. The experimentally measured solutions are indicated by filled black circles with error bars in Fig. 3.

For example, in region (vii) at $f_{b}=6.35 \mathrm{kHz}$ a solution with seven dips emerges from the interference introduced at the boundaries [see Fig. 4(a)]. However, as we gradually increase the frequency, the outermost dips approach the boundaries until the solution collides and vanishes in a saddle-node bifurcation with an intermediate seven-dip solution, i.e., the second lowest branch shown in region (vii) in Fig. 3. As a result of the disappearance of this branch, the lowest branch in region (v) is made up of solutions with only five dips, e.g. for $f_{b}=6.80 \mathrm{kHz}$ [see Fig. 4(b)]. The cascade of saddle-node bifurcations continues as we gradually increase the frequency. A solution with only three dips emerges in region (iii), e.g., for $f_{b}=7.15 \mathrm{kHz}$ [see Fig. 4(c)]. Finally, a single-dip DB emerges for frequencies in region (i), e.g., for $f_{b}=7.29 \mathrm{kHz}$ (see Fig. 1). Thus, we conclude that the actuation frequency that is chosen will dictate ("dial in") the number of dips that will emerge upon actuation.

\section{A. Experimental observation of bistability}

In addition to controlling the multibreather via the frequency, one can control the number of dips of the DB by varying the amplitude. Consider, for example, the three solution branches shown in region (iii) in Fig. 3 at $f_{b}=7.14 \mathrm{kHz}$. At this frequency the system is bistable, with the three-dip and single-dip breathers being stable and an intermediate three-dip solution being unstable. These three solutions were continued in amplitude [see Fig. 5(a)], revealing a canonical hysteresis loop between the stable three-dip and the single-dip solution branches. This enables, even experimentally, a jump between the different solution types [as shown in Fig. 5(b)]. One can excite the single-dip breather in the parameter region $a \in(0.12,0.37)$ by first driving to the single-dip breather with $a>0.37$ and then adjusting the amplitude to some value in the region $a \in(0.12,0.37)$ [see Fig. 5(d) and 5(e), for example]. Note that exciting a resting chain for a fixed frequency in that region will yield the three-dip breather [see, e.g., Fig. 5(b)], a feature indicative of the system's bistability.

\section{LINEAR STABILITY ANALYSIS}

To investigate the dynamical stability of the obtained states, a Floquet analysis was carried out to compute the multipliers associated with the DBs. The Floquet multipliers for a solution were obtained by computing the eigenvalues of the monodromy matrix [which is $V\left(T_{b}\right)$ upon convergence of the Newton scheme]. We focus on instabilities associated with saddle-node bifurcations and pitchfork bifurcations; therefore, we are chiefly interested in the Floquet multipliers on the (positive) real line. However, there can also be oscillatory instabilities, which correspond to complex-conjugate pairs of Floquet multipliers lying outside of the unit circle. Figure 6 shows examples of the Floquet multipliers for three single-dip solutions. The first two examples, in Figs. 6(a) and 6(b), are unstable, but the third example, in Fig. 6(c), is asymptotically stable. A plot of the magnitude of the multipliers for fixed $a=0.2 \mu \mathrm{m}$ and various $f_{b}$ is shown in Fig. 6(d). A complication revealed by the Floquet analysis is that, even though asymptotically stable solutions are possible, the damping is weak enough that these solutions may not be realizable in the 10-ms window considered experimentally. For example, for $f_{b}=7.29 \mathrm{kHz}$ and $a=0.2 \mu \mathrm{m}$ there is a DB with Floquet multipliers in the interval $|\lambda| \in[0.974,0.998]$, and thus it is asymptotically stable. Because these multipliers are so near the unit circle, converging to a fixed point by repeatedly applying $F$ to some initial condition, which is analogous to what happens when the experiment is run for multiple periods, requires a large number of iterations or running an experiment 

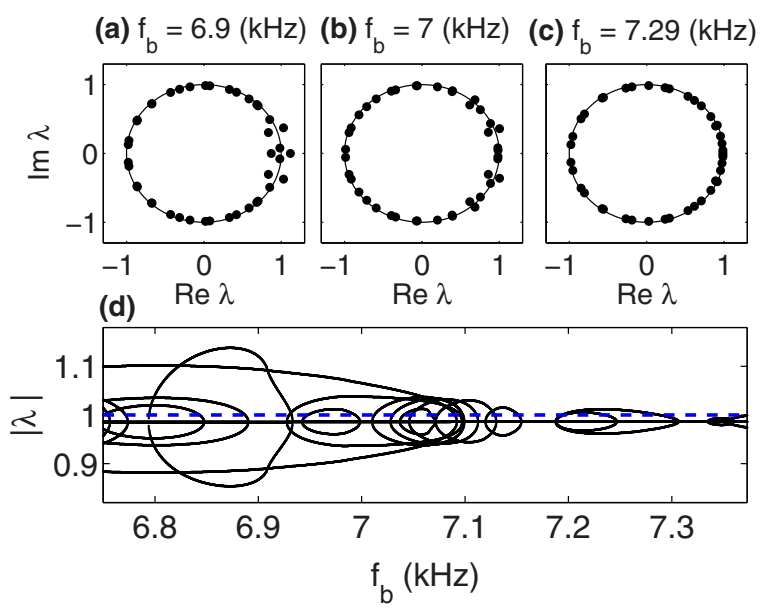

FIG. 6. (Color online) Floquet multipliers of the single-dip dark breather for $a=0.2 \mu \mathrm{m}$. (a) At $f_{b}=6.90 \mathrm{kHz}$ the solution is unstable due to oscillatory instabilities as well as Floquet multipliers on the real line. (b) Floquet multipliers on the real line have retreated within the unit circle at $f_{b}=7.00 \mathrm{kHz}$ but the oscillatory instabilities remain. (c) Finally, at $f_{b}=7.29 \mathrm{kHz}$ all instabilities have vanished, and the solution is asymptotically stable. (d) Magnitude of Floquet multipliers for frequencies $(\mathrm{kHz})$ in the interval $f_{b} \in(6.75,7.4)$. A dashed (blue) line at $|\lambda|=1$ is also shown to help identify regions of asymptotic stability.

for an extended period of time. Therefore, due to the 10-ms window used (experimentally), we are often only able to see the experiment approach the DB and do not see it fully converge to the DB. Conversely, there also exist oscillatory instabilities on the solution branch for some parameter intervals. Although the DB is now unstable, we do not expect an oscillatory instability to manifest itself in the 10-ms window if we start with an initial condition near enough to the unstable DB. In that case, we have found that the effects of the oscillatory instability are typically not observable in computations until approximately $t=300 \mathrm{~ms}$. The reason for this is that the magnitudes of the Floquet multiplier pairs associated with the oscillatory instabilities are often closer to unity than the magnitudes of the unstable Floquet multipliers on the real line. For this reason we only indicate the instabilities due to Floquet multipliers on the real line [dashed (red) lines] in Fig. 3, even though oscillatory instabilities may also be present. A solid (blue) line is shown otherwise, indicating the absence of a real instability.

\section{A DEEPER THEORETICAL PROBING OF THE BIFURCATIONS}

The bifurcation diagram in Fig. 3 provides a simple yet powerful qualitative description of the breather structures that can emerge in a monatomic granular chain. However, as the above Floquet analysis suggests, there is a deeper structural complexity that has important implications for the interpretation of the experimental findings. In particular, there are apparently few regions where the main branch is asymptotically stable (see, e.g., Fig. 6). This begs the question, "Why do the solutions making up the main branch emerge upon exciting a resting chain?" In this section, we unveil several subtleties of the bifurcation structure of this system, hoping
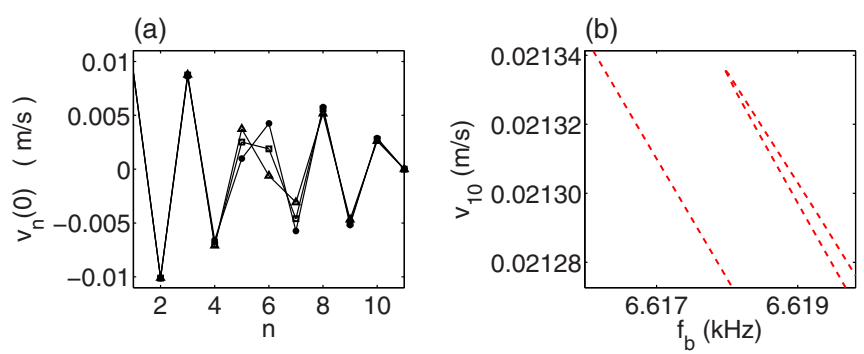

FIG. 7. (Color online) (a) Velocity profile for three variants of a three-dip breather for $a=0.2 \mu \mathrm{m}$ and $f_{b}=6.62 \mathrm{kHz}$, indicated by squares, circles, and triangles at $t=0$. Since the solutions have odd symmetry with respect to the center bead $(n=11)$, only the left part of the chain is shown. (b) Tight zoom of Fig. 3, where three distinct (but connected) branches can be seen (which cannot be discerned in the former figure). The three solutions shown in panel (a) lie on the branches shown in panel (b).

to at least partially explain the answer to this question. One subtlety that is not apparent in Fig. 3 is how dense the coils are. For example, a "zoomed-in" version of one of the coils, shown in Fig. 7(b), reveals that the branch actually coils several times in tight regions in parameter space. Another finer detail is that the primary branch shown in Fig. 3 generates many secondary and tertiary branches of solutions. Figure 8 shows a "zoomed-out" version of the primary branch, indicating that the coiling structure persists well below the $6-\mathrm{kHz}$ frequency level that was studied experimentally. The (cyan) diamonds, (green) squares, and black asterisks indicate the presence of (a) pitchfork, (b) period-doubling, and (c) Neimark-Sacker bifurcation points, respectively. The former two are the points on the main branch where the secondary branches of periodic solutions are born, whereas the Neimark-Sacker points suggest the existence of quasiperiodic solutions. We briefly describe scenarios (a)-(c) below.

a. Pitchfork bifurcations off the main branch. Several secondary branches are initiated by pitchfork bifurcations, either due to a regular pitchfork bifurcation or as part of a pair in what we call a pitchfork loop. To illustrate the difference, we present an example of each type in Fig. 9. The pitchfork loop, shown in Fig. 9(a), consists of a pair of pitchfork bifurcations that are connected. These pitchfork loops appear to be relatively short-lived; i.e., the pitchfork bifurcation that "opens" the loop is near (in terms of arc length) to the pitchfork bifurcation that "closes" the loop. As a result, although the main branch may have an unstable Floquet multiplier on the real line, there always appears to be a pair of "nearby" solutions that are qualitatively similar to the main branch, with the addition of a small component that breaks the symmetry that solutions have on the main branch. However, the two secondary solutions that comprise the pitchfork loop appear to be symmetric to each other; the only difference between the branches is the direction in which the symmetry-breaking component manifests itself. An example of this is shown in Fig. 10. The velocity profile, which is an odd function on the main branch if the center bead is taken as the origin, loses this symmetry on either branch of the pitchfork loop. This should be contrasted with the "tightly coiled" solutions in Fig. 7, which are perturbations of one another but still have velocity 


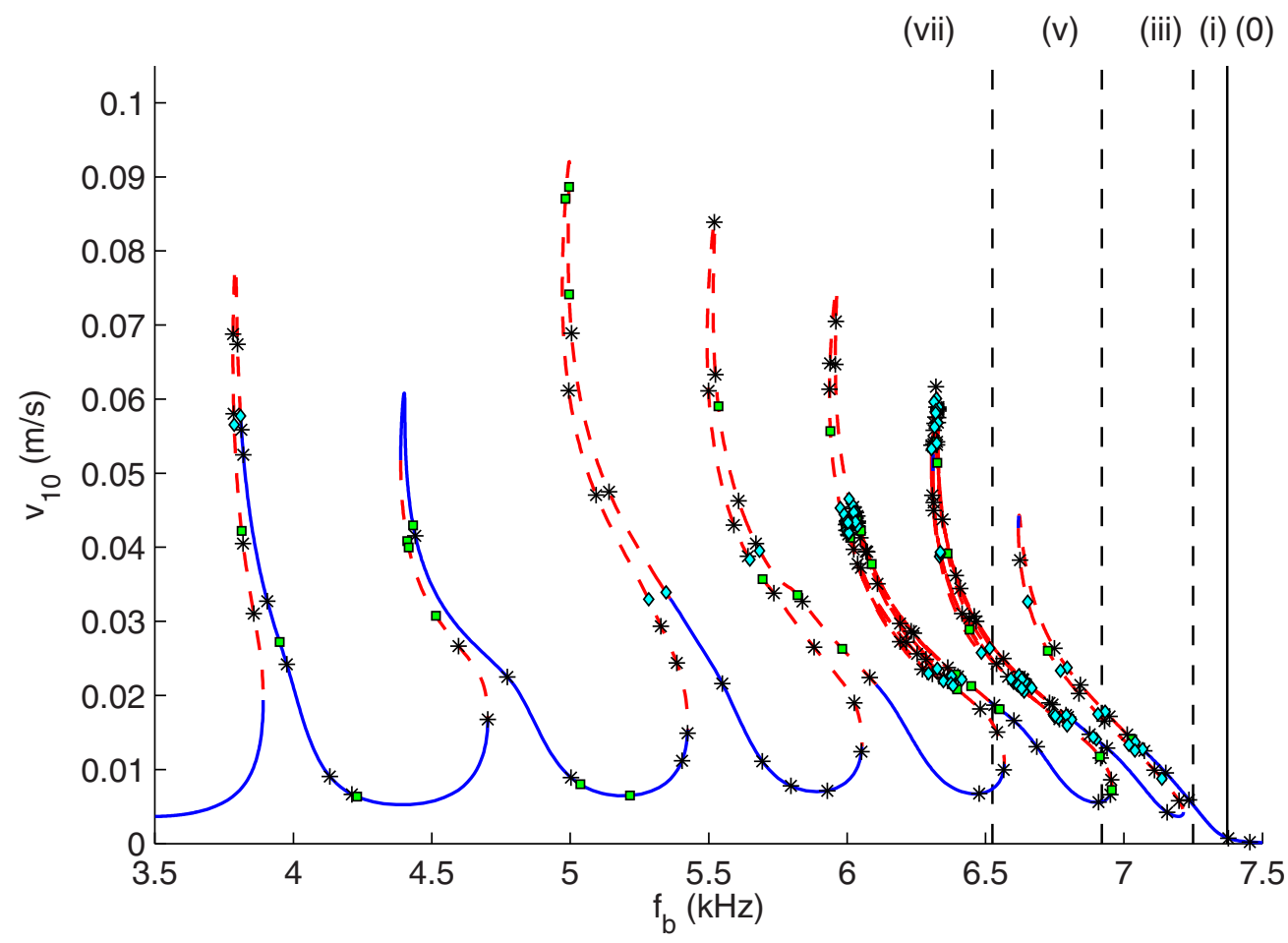

FIG. 8. (Color online) An extended illustration of the branch of solutions shown in Fig. 3. As in Fig. 3, the solid (blue) curves indicate that the real Floquet multipliers are within (or on) the unit circle. A dashed (red) line is shown otherwise. Symbols indicate the presence of a bifurcation: black asterisks are Neimark-Sacker bifurcations to $T^{2}$ tori, (green) squares are period-doubling bifurcations, and (cyan) diamonds are pitchfork bifurcations. Here, we have plotted only half the total number of torus bifurcations, to avoid obscuring the plot. Dashed lines indicate regions where the $N$-dip solutions appear as shown in Fig. 3.

profiles that are odd functions, again using the center bead as the origin. There are also regular pitchfork bifurcations that produce solution branches that do not quickly "close." We refer to the secondary branches created by these bifurcations as pitchfork branches and show an example of one in Fig. 9(b). Both types of pitchfork bifurcations stemming from the main branch that we studied were unstable for most of the values of $f_{b}$ and $v_{10}$ that were studied experimentally.

(a)

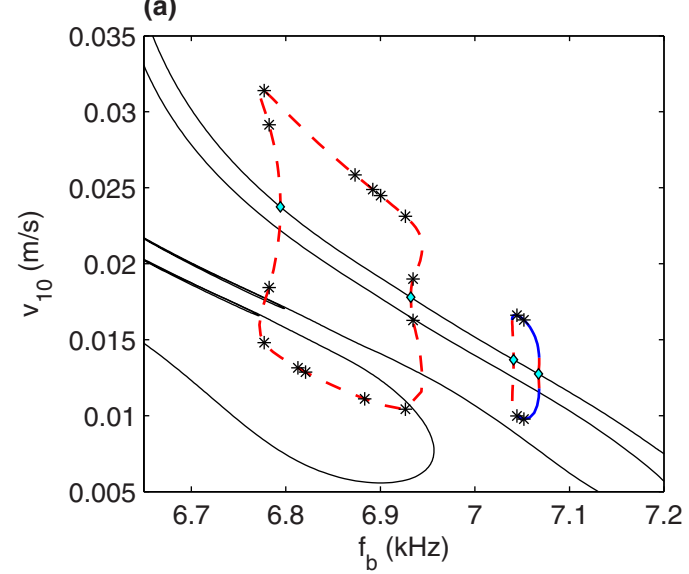

b. Period-doubling bifurcations off the main branch. Perioddoubling bifurcations are another source of asymmetric periodic orbits. As indicated in Fig. 8 by the (green) squares, there is a number of secondary period 2 branches created by the main branch of DBs (which are period 1). In each of the examples we found, the eigenvector with $\lambda=-1$ is an even function, which breaks the odd symmetry that is present on the main branch of solutions. A prototypical example of a secondary,

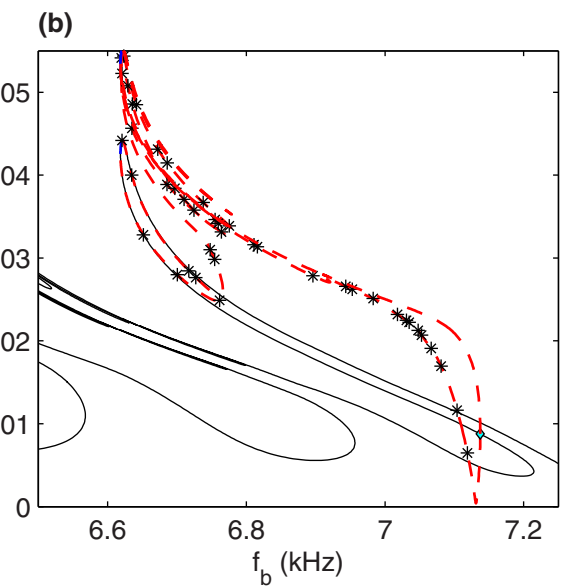

FIG. 9. (Color online) Examples of secondary branches with torus bifurcations indicated by black asterisks and pitchfork bifurcations indicated by (cyan) diamonds. As in Fig. 3, solid (blue) curves indicate that the real Floquet multipliers are within (or on) the unit circle. A dashed (red) line is shown otherwise. The main solution branch is shown in black. (a) Plot of two loops, each of which consists of two pitchfork bifurcations. (b) Plot of pitchfork branches that are not part of a pitchfork loop. 

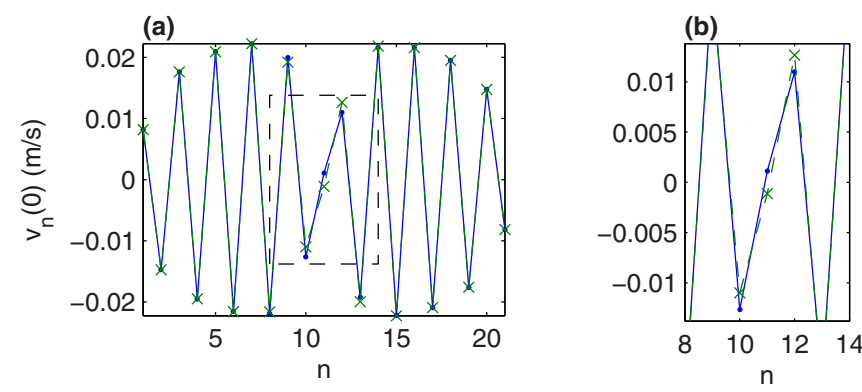

FIG. 10. (Color online) (a) Plot of the velocity profiles at $t=0$ on either "arm" of the pitchfork loop shown in the inset in Fig. 3, which demonstrate the symmetry breaking that occurs when the forcing amplitude is at a maximum. The solid (blue) line is the lower branch, while the dashed (green) line is the upper branch. (b) Zoom-in of (a) around beads 8-14.

period 2 branch is shown in Fig. 11. This period 2 branch acts as a "bridge" between two sides of a single "lobe" of period 1 solutions as indicated by the pair of pitchfork bifurcations at the start and end of the period 2 branch of solutions (each bifurcation is indeed a pitchfork, but the ordinate in Fig. 11, which is the maximum velocity of the tenth bead, causes two branches of the pitchfork to appear practically identical). As in the period 1 branch, the period 2 branch contains its own set of bifurcations including additional branch points, period-doubling bifurcations, and Neimark-Sacker bifurcations, each of which could create tertiary period 2, period 4, and quasiperiodic solutions, respectively. However, none of the tertiary period 2 or period 4 branches we examined was born stable at their associated bifurcation points, nor was stability regained in the tertiary branch. Although our search of these regions is less than exhaustive, the (quite common) tertiary branches stemming from period 2 branches we computed were unstable.

c. Quasiperiodic solutions. Within the framework of classical dynamical systems theory, one would expect quasiperiodic
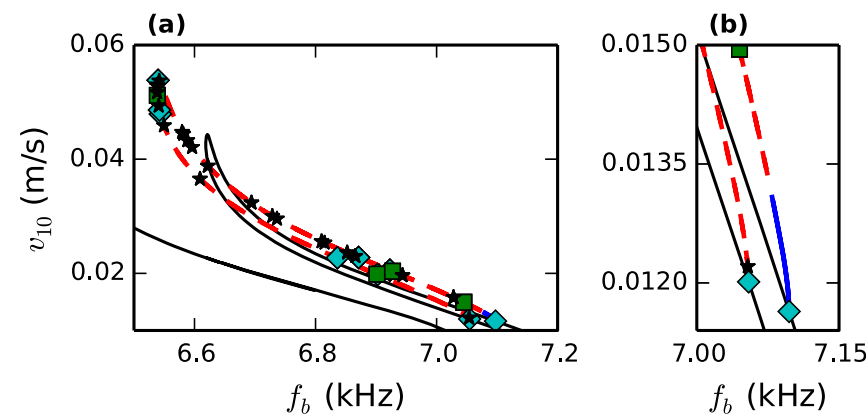

FIG. 11. (Color online) (a) Example of a period 2 branch of solutions is shown in blue and red; solid (blue) curves indicate that the real Floquet multipliers are within (or on) the unit circle. A dashed (red) line is shown otherwise. The black curve denotes the period 1 branch of solutions, and the markers indicate pitchfork bifurcation [(cyan) diamonds], period-doubling bifurcations [(green) squares], and Neimark-Sacker bifurcations (black asterisks). (b) A zoom-in of (a) showing how each end of the period 2 branch is connected to the main branch by a pair of pitchfork bifurcations. solutions to exist in the system given the presence of NeimarkSacker points. A quasiperiodic solution is a continuous time trajectory on a torus in phase space; in the stroboscopic map, that same quasiperiodic solution appears as an invariant circle. Analogous to the periodic orbits generated by Hopf bifurcations, Neimark-Sacker bifurcations produce invariant circles that can be either stable or unstable. While algorithms for approximating invariant circles exist [44], continuing branches of quasiperiodic orbits via Newton's method lies outside the scope of this paper. However, in the neighborhood of a supercritical Neimark-Sacker bifurcation, it is possible to approximate the invariant circle by iterating the stroboscopic map a large number of times as demonstrated in Fig. 12. Clearly, such an approach is only feasible when the invariant circle (or, in a flow, the torus) is asymptotically stable, as the rate of convergence is dependent upon the "least stable" eigenvalue. As such, Fig. 12 shows two examples on the same "branch" of solutions obtained by computing $10^{5}$ successive iterates of the stroboscopic map for each new value of $f_{b}$ and then plotting the next 5000-10 000 iterates of the stroboscopic map, with one marker every 10 iterates. At $f_{b}=7.487 \mathrm{kHz}$, we have identified what appears to be a quasiperiodic orbit as shown in Figs. 12(a) and 12(b). Figure 12(a) shows the displacement and velocity of the tenth bead at the start of every forcing period. Smaller (blue) dots denote the state of the system (in this projection) after every 10 iterates of the stroboscopic map, while filled (green) circles denote the last 20 iterates of the map; finally, the filled (red) circle in the center is the period 1 solution at this frequency. Although the presence of an invariant circle is clear in this projection, it should be noted that it requires several thousand iterations to reach the vicinity of the invariant circle given an initial condition that is a small perturbation of the period 1 solution. This is due to the fact that we are near the Neimark-Sacker bifurcation point, so the unstable pair of eigenvalues is only slightly larger than unity in magnitude. However, the invariant circle itself appears to be stable even in the face of large perturbations. Figure 12(b) shows the relative distance between two nearby trajectories as a function of the iterations of the stroboscopic map. To produce this plot each (nondimensionalized) component of a point on the invariant circle was perturbed with a small "kick" drawn from a normal distribution with a standard deviation of $10^{-5}$, which is small compared to typical, nondimensionalized displacement and velocities at this forcing frequency. The main result is that points that are not too far apart will converge as the perturbed solution is attracted back to the invariant circle and will remain close for all future times. This behavior should be contrasted with what occurs at $f_{b}=7.462$ $\mathrm{kHz}$, which is shown in Figs. 12(c) and 12(d). Although the dynamics shown in Fig. 12(c) appear to lie on a higher dimensional torus, say, a $T^{3}$ torus, the plot of the distance between nearby initial conditions in Fig. 12(d) indicates that we are more likely on a chaotic attractor, as nearby trajectories diverge after sufficiently long periods of time. This can be explained by the Ruelle-Takens-Newhouse route to chaos, which is based on the observation that a constant vector field on a $T^{3}$ torus can be perturbed by an arbitrarily small amount to produce a chaotic attractor [45]. As a result, the quasiperiodic solution at $f_{b}=7.487 \mathrm{kHz}$ likely underwent another transition at some higher frequency, which results 
(a)

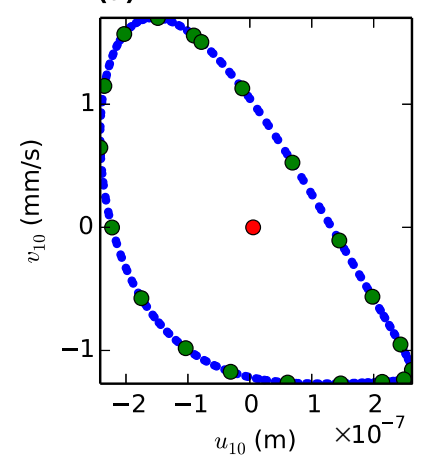

(b)

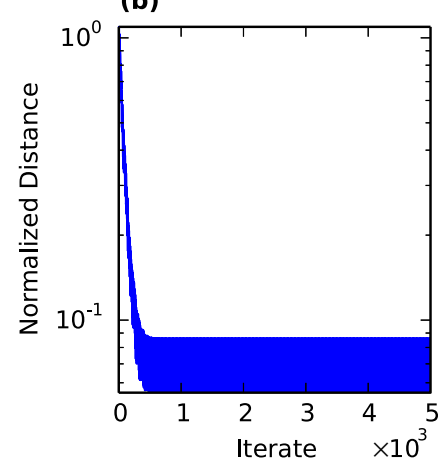

(c)

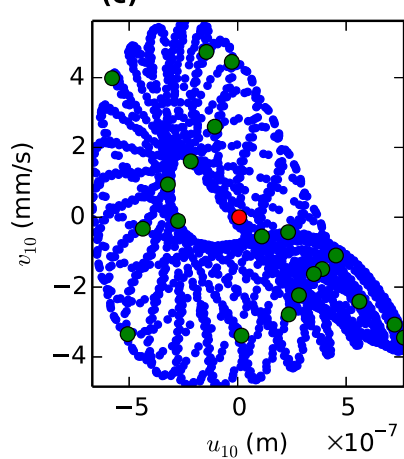

(d)

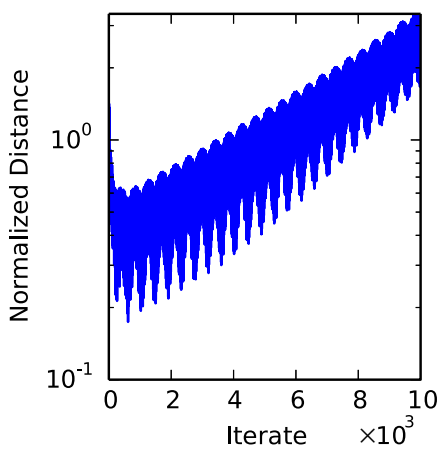

FIG. 12. (Color online) (a) Demonstration of the invariant circle created by plotting several thousand iterates of the map $F$ with $f_{b}=$ $7.487 \mathrm{kHz}$; smaller (blue) dots indicate the velocity of the tenth bead recorded every $10 / f_{b}$ s, filled (green) circles denote the last 20 iterates of the map, and the filled (red) circle in the center denotes the unstable period 1 orbit. (b) The log of the normalized distance between two nearby initial conditions as a function of time; though the exact distance is time dependent, trajectories on the invariant circle that start near each other remain near each other. (c) Demonstration of the stroboscopic map with $f_{b}=7.462$ colored as in the leftmost plot; if more iterations are plotted, the trajectory will eventually "fill in" the region. (d) Pairs of nearby trajectories will separate after a sufficiently large number of iterations, which suggests the presence of a chaotic attractor.

in the observed dynamics at $7.462 \mathrm{kHz}$. We should point out that not all of the Neimark-Sacker bifurcations in Fig. 8 result in stable quasiperiodic orbits. In principle, subcritical Neimark-Sacker bifurcations will produce unstable invariant circles that cannot be continued using the straightforward computational procedure used to produce Fig. 12 but could be continued using more sophisticated techniques like those in Ref. [44].

\section{A. Implication of secondary structures for experiments}

Due to the number of bifurcations that generate secondary branches and the complexity and additional bifurcations that appear on those branches, this system apparently gives rise to a veritable "zoo" of dynamics and displays a vast array of different nonlinear behaviors. To complicate matters further, there are also additional branches of periodic solutions with longer periods (e.g., period 2 solutions of the map $F$ that have period $2 T_{b}$ ) whose shapes and bifurcations are also nontrivial. Both these larger period solutions and solutions on the pitchfork branches generate tertiary branches, which, in principle, could be asymptotically stable in the region of experimental interest but, in our experience, are typically unstable. As shown in Fig. 8 and Fig. 9, the main branch and the secondary branches are filled with Neimark-Sacker bifurcations. This gives many opportunities for the creation of quasiperiodic solution branches, where the dynamics lies on an invariant circle in the stroboscopic map (and a torus in the flow). Thus, it is possible that some of the solutions observed experimentally actually lie on or near a slowly modulated torus (which live near the main branch of period 1 DBs). However, observing the true quasiperiodic nature of these solutions experimentally may be difficult; in the numerical study performed here, thousands of forcing periods were required before the quasiperiodic structure of the solution became clear. Our answer to the question posed at the beginning of this section is as follows: There is a very rich variety of periodic solutions that exist. There are additional nearby invariant objects (tori, chaotic solutions) and long transients associated with global bifurcations that also "lurk" in the neighborhood of these solutions. Thus, a trajectory starting from zero initial data is highly nontrivial and appears to be influenced by the zoo of structures that exist in parameter space. Indeed, one is often not completely certain that a "visually close to periodic" transient has actually converged to a limit cycle on a particular branch. Ultimately, our comparison of theory and experiment suggests that it is possible to obtain a good qualitative description of the observed dynamics based on the main branch of period 1 solutions. This was true even in unstable regions, probably due to the existence of similar structures that live near the main branch of period 1 solutions, which, given our investigations, are plentiful.

\section{CONCLUSIONS AND FUTURE CHALLENGES}

The damped-driven granular crystal system has been shown to provide access to a rich family of DB and multibreather solutions. In fact, it can be argued that the resulting mapping of the system's solutions appears to be far richer than what has been previously explored for any breather structure in granular chains (and, in the context of DBs, for any physical system). The system possesses an intricate bifurcation diagram where the stable single- and multidip solutions are interlaced via unstable intermediate branches (i.e., snaking behavior). The diagram contains a large number of saddle-node bifurcations and associated fold points, as well as pitchfork symmetry-breaking points. This structure provides not only hysteresis loops and multistability regimes, but also a remarkable tunability and numerous branches of more complex period 2, quasiperiodic, and even chaotic attractor solutions. We have offered, through our dynamical systems analysis, a roadmap towards the identification of these solutions, although some of them (especially the more complex ones, including quasiperiodic and chaotic solutions) clearly merit further investigation. The selection of driving frequency and amplitude enables a selection of multibreather configurations robustly sustained by the dynamics. The coherent structures produced herein 

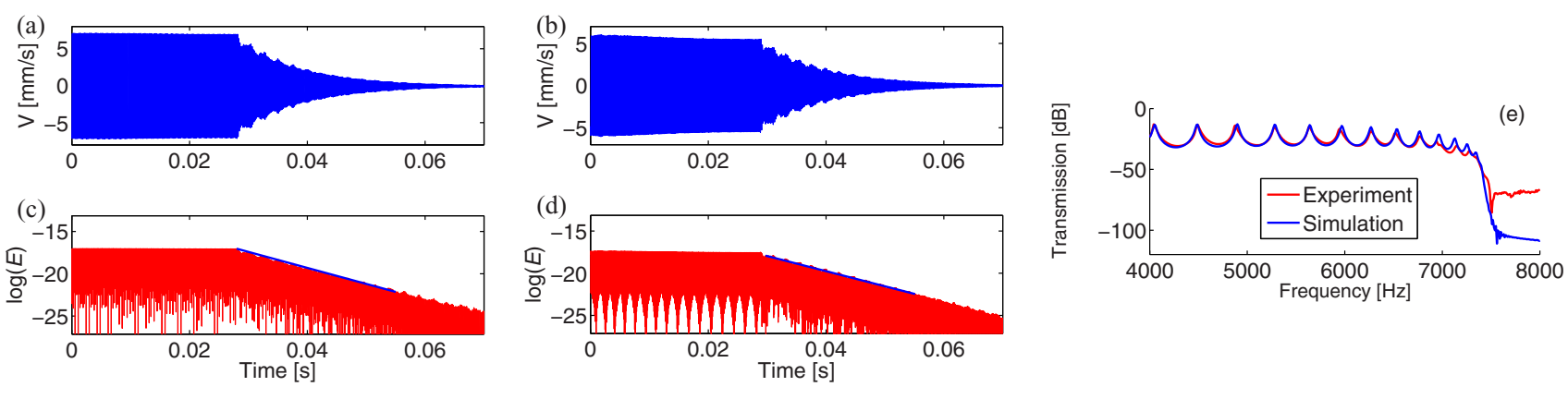

FIG. 13. (Color online) Temporal profiles of the 21st particle's velocity and kinetic energy. (a, c) Experimental measurements. (b, d) Numerical simulations. (e) Experimental (red line) and numerical (blue line) results of transmission gains of the granular chain.

could be utilized towards controllable energy funneling and harvesting within granular media.

\section{ACKNOWLEDGMENTS}

The authors would like to thank G. Theocharis for useful discussions. Support from the US NSF (Grant Nos. CMMI 1310173, CMMI 1234452, and CMMI 1000337) and US AFOSR (Grant No. FA9550-12-1-0332) is appreciated. M.O.W. gratefully acknowledges support from an NSF Mathematics Sciences Postdoctoral Research Fellowship, DMS 1204783.

\section{APPENDIX: DAMPING COEFFICIENT DETERMINATION}

The damping mechanism in the granular system is modeled by the simple mathematical term $\frac{M}{\tau} \dot{u}_{n}$, as expressed in Eq. (1). Despite its simplicity, this dash-pot model has proven to be effective in granular systems as reported in previous studies, including the authors' recent work [22,27]. The damping coefficient $\tau$ is determined empirically to reflect the degree of dissipation in the given experimental system. More specifically, we excite the chain with harmonic pulses, with $a=0.03 \mu \mathrm{m}$ and $f=4.815 \mathrm{kHz}$, and then quench the excitation at $\approx 28 \mathrm{~ms}$. The temporal velocity profile of the 21st particle (i.e., the last particle in the chain) is shown in Fig. 13(a) based on the measurements with a laser Doppler vibrometer. Based on this velocity profile, Fig. 13(c) plots the decay of the kinetic energy in logarithmic scale. We observe that the slope of the kinetic energy decay is linear after $28 \mathrm{~ms}$. This, in turn, leads to the empirical value of $\tau \approx 5 \mathrm{~ms}$ based on the fitting of this decaying slope [blue line in Fig. 13(c)]. We validate this value by reproducing the decaying curves numerically [Figs. 13(b) and 13(d)]. The simulation results are in excellent agreement with the experimental results. Since the aforementioned damping factor was determined at a single excitation frequency, we need to check the efficacy of this damping model over a broad range of the frequency domain. First, we obtain the experimental baseline of the system's frequency response by measuring its transmission gain in terms of the power spectral density using a network analyzer (Agilent 4395A). Then we calculate its numerical counterpart by solving the differential equation of the system [Eq. (1)] using the empirically obtained damping parameter. The experimental and numerical results are plotted in Fig. 13(e) by the red and blue curves, respectively. The numerical results successfully capture the dynamic response of the system over the broad range of the pass band, confirming the validity of our simple damping model.
[1] S. Flach and A. V. Gorbach, Phys. Rep. 467, 1 (2008).

[2] F. Lederer, G. I. Stegeman, D. N. Christodoulides, G. Assanto, M. Segev, and Y. Silberberg, Phys. Rep. 463, 1 (2008).

[3] M. Sato, B. E. Hubbard, and A. J. Sievers, Rev. Mod. Phys. 78, 137 (2006).

[4] P. Binder, D. Abraimov, A. V. Ustinov, S. Flach, and Y. Zolotaryuk, Phys. Rev. Lett. 84, 745 (2000); E. Trías, J. J. Mazo, and T. P. Orlando, ibid. 84, 741 (2000).

[5] L. Q. English, M. Sato, and A. J. Sievers, Phys. Rev. B 67, 024403 (2003); U. T. Schwarz, L. Q. English, and A. J. Sievers, Phys. Rev. Lett. 83, 223 (1999).

[6] B. I. Swanson, J. A. Brozik, S. P. Love, G. F. Strouse, A. P. Shreve, A. R. Bishop, W.-Z. Wang, and M. I. Salkola, Phys. Rev. Lett. 82, 3288 (1999).

[7] M. Peyrard, Nonlinearity 17, R1 (2004).

[8] O. Morsch and M. Oberthaler, Rev. Mod. Phys. 78, 179 (2006).
[9] A. Chabchoub, O. Kimmoun, H. Branger, N. Hoffmann, D. Proment, M. Onorato, and N. Akhmediev, Phys. Rev. Lett. 110, 124101 (2013).

[10] A. Weller, J. P. Ronzheimer, C. Gross, J. Esteve, M. K. Oberthaler, D. J. Frantzeskakis, G. Theocharis, and P. G. Kevrekidis, Phys. Rev. Lett. 101, 130401 (2008); S. Stellmer, C. Becker, P. Soltan-Panahi, E.-M. Richter, S. Dörscher, M. Baumert, J. Kronjäger, K. Bongs, and K. Sengstock, ibid. 101, 120406 (2008).

[11] D. J. Frantzeskakis, J. Phys. A 43, 213001 (2010).

[12] W. Tong, M. Wu, L. D. Carr, and B. A. Kalinikos, Phys. Rev. Lett. 104, 037207 (2010).

[13] A. Kanshu, C. Rüter, D. Kip, J. Cuevas, and P. G. Kevrekidis, Eur. Phys. J. D 66, 182 (2012).

[14] V. F. Nesterenko, Dynamics of Heterogeneous Materials (Springer-Verlag, New York, 2001). 
[15] S. Sen, J. Hong, J. Bang, E. Avalos, and R. Doney, Phys. Rep. 462, 21 (2008).

[16] C. Daraio, V. F. Nesterenko, E. B. Herbold, and S. Jin, Phys. Rev. Lett. 96, 058002 (2006).

[17] J. Hong, Phys. Rev. Lett. 94, 108001 (2005).

[18] F. Fraternali, M. A. Porter, and C. Daraio, Mech. Adv. Mat. Struct. 17(1), 1 (2010).

[19] R. Doney and S. Sen, Phys. Rev. Lett. 97, 155502 (2006).

[20] D. Khatri, C. Daraio, and P. Rizzo, SPIE 6934, 69340U (2008).

[21] A. Spadoni and C. Daraio, Proc. Natl. Acad. Sci. USA 107, 7230 (2010).

[22] N. Boechler, G. Theocharis, and C. Daraio, Nature Mater. 10, 665 (2011).

[23] C. Daraio, V. F. Nesterenko, E. B. Herbold, and S. Jin, Phys. Rev. E 72, 016603 (2005).

[24] V. F. Nesterenko, C. Daraio, E. B. Herbold, and S. Jin, Phys. Rev. Lett. 95, 158702 (2005).

[25] G. Theocharis, M. Kavousanakis, P. G. Kevrekidis, C. Daraio, M. A. Porter, and I. G. Kevrekidis, Phys. Rev. E 80, 066601 (2009); S. Job, F. Santibanez, F. Tapia, and F. Melo, ibid. 80, 025602 (2009); Y. Man, N. Boechler, G. Theocharis, P. G. Kevrekidis, and C. Daraio, ibid. 85, 037601 (2012).

[26] N. Boechler, G. Theocharis, S. Job, P. G. Kevrekidis, M. A. Porter, and C. Daraio, Phys. Rev. Lett. 104, 244302 (2010); G. Theocharis, N. Boechler, P. G. Kevrekidis, S. Job, M. A. Porter, and C. Daraio, Phys. Rev. E 82, 056604 (2010).

[27] C. Hoogeboom, Y. Man, N. Boechler, G. Theocharis, P. G. Kevrekidis, I. G. Kevrekidis, and C. Daraio, Eur. Phys. Lett. 101, 44003 (2013).
[28] C. Chong, P. G. Kevrekidis, G. Theocharis, and C. Daraio, Phys. Rev. E. 87, 042202 (2013).

[29] G. Schneider, Appl. Anal. 89, 1523 (2010).

[30] A. Alvarez, J. F. R. Archilla, J. Cuevas, and F. R. Romero, New J. Phys. 4, 72 (2002).

[31] G. James, J. Nonlin. Sci. 13, 27 (2003).

[32] B. Sanchez-Rey, G. James, J. Cuevas, and J. F. R. Archilla, Phys. Rev. B 70, 014301 (2004).

[33] http://www.efunda.com.

[34] F. Li, L. Yu, and J. Yang, J. Phys. D: Appl. Phys. 46, 155106 (2013).

[35] J. Burke and E. Knobloch, Chaos 17, 037102 (2007).

[36] J. H. P. Dawes, SIAM J. Appl. Dyn. Syst. 7, 186 (2008).

[37] S. G. McCalla and B. Sandstede, Physica D 239, 1581 (2010).

[38] A. Bergeon, J. Burke, E. Knobloch, and I. Mercader, Phys. Rev. E 78, 046201 (2008).

[39] J. Knobloch, D. J. B. Lloyd, B. Sandstede, and T. Wagenknecht, J. Dyn. Diff. Eqs. 23, 93 (2011).

[40] M. Beck, J. Knobloch, D. J. B. Lloyd, and B. T. Sandstede Wagenknecht, SIAM J. Math. Anal. 41, 936 (2009).

[41] C. Taylor and J. H. P. Dawes, Phys. Lett. A 375, 14 (2010).

[42] F. Haudin, R. G. Rojas, U. Bortolozzo, S. Residori, and M. G. Clerc, Phys. Rev. Lett. 107, 264101 (2011).

[43] T. M. Schneider, J. F. Gibson, and J. Burke, Phys. Rev. Lett. 104, 104501 (2010).

[44] I. G. Kevrekidis, R. Aris, L. D. Schmidt, and S. Pelikan, Physica D 16, 243 (1985).

[45] S. E. Newhouse, D. Ruelle, and F. Takens, Commun. Math. Phys. 64, 35 (1978). 